\title{
Warp-speed adaptation to novel hosts after 300 generations of enforced dietary specialisation in the seed beetle Callosobruchus maculatus (Coleoptera: Chrysomelidae: Bruchinae)
}

\author{
Thomas N. PRICE ${ }^{1,2}$, Aoife LEONARD ${ }^{1}$ and LeSLey T. LANCASTER ${ }^{1, *}$ \\ ${ }^{1}$ Institute of Biological and Environmental Sciences, Zoology Building, University of Aberdeen, Aberdeen AB24 2TZ, UK; \\ e-mails: t.price.12@aberdeen.ac.uk,r05al15@abdn.ac.uk, lesleylancaster@abdn.ac.uk \\ ${ }^{2}$ Current address: Centre for Ecology and Conservation, College of Life and Environmental Sciences, University of Exeter, \\ Cornwall Campus, Penryn TR10 9EZ, UK
}

\begin{abstract}
Key words. Coleoptera, Chrysomelidae, Bruchinae, Callosobruchus maculatus, emerging crop pests, host shifts, genetic variation, adaptation trajectory, evolvability, experimental evolution, quasi-natural selection, heritability
\end{abstract}

\begin{abstract}
Herbivorous insects are often highly specialised, likely due to trade-offs in fitness on alternative host species. However, some pest insects are extremely adaptable and readily adopt novel hosts, sometimes causing rapid expansion of their host range as they spread from their original host and geographic origin. The genetic basis of this phenomenon is poorly understood, limiting our ability to predict or mitigate global insect pest outbreaks. We investigated the trajectory of early adaptation to novel hosts in a regionally-specialised global crop pest species (the cowpea seed beetle Callosobruchus maculatus). After experimentallyenforced dietary specialisation for nearly 300 generations, we measured changes in fitness over the first 5 generations of adaptation to 6 novel hosts. Of these, C. maculatus reproduced successfully on all but one, with reduced fitness observed on three hosts in the first generation. Loss of fitness was followed by very rapid, decelerating increases in fitness over the first 1-5 generations, resulting in comparable levels of population fitness to that observed on the original host after 5 generations. Heritability of fitness on novel hosts was high. Adaptation occurred primarily via changes in behavioural and phenological traits, and never via changes in offspring survival to adulthood, despite high heritability for this trait. These results suggest that $C$. maculatus possesses ample additive genetic variation for very rapid host shifts, despite a prolonged period of enforced specialization, and also suggest that some previously-inferred environmental maternal effects on host use may in part actually represent (rapidly) evolved changes. We highlight the need to examine in more detail the genetic architecture facilitating retention of high additive genetic variation for host shifts in extremely adaptable global crop pests.
\end{abstract}

\section{INTRODUCTION}

Herbivorous insect distributions are generally constrained by the geographic range of their host plants, and this relationship is often highly specialised (Koizumi et al., 1999; Scriber, 2002). Extreme specialisation of host use among herbivorous insects is thought to reflect strong fitness trade-offs associated with alternative host plant use (Joshi \& Thompson, 1995). However, a minority of herbivorous insects are generalists capable of utilising multiple hosts or of undergoing rapid host shifts as their conditions change. These species are particularly detrimental to human food security when they adapt to feed on a range of economically important agricultural products. With the industrialisation of agriculture, and the rapid transport of both plant species and the insects themselves, rapid and widespread adaptation to novel hosts is becoming increas- ingly common in many herbivorous pest insects (Bowers et al., 1992; Gutierrez \& Thomas, 2000; Stastny et al., 2006). Under this process of rapid host range expansions and shifts, herbivorous insects have come to represent a major component of all agricultural pest species in the tropical and sub-tropical world, and these are now spreading globally to more temperate regions as well (Risch et al., 1983; Bebber et al., 2013; Lancaster, 2016). To facilitate transitions to additional or alternative agricultural hosts, herbivorous insects must often undergo evolutionary adaptation in two aspects of their biology. These are behavioural changes in oviposition by females and physiological changes in larva's ability to use the novel host as a food source (Jaenike, 1990). Both behavioural and physiological shifts are often based on substantial genetic changes (Wasserman \& Futuyma, 1981; Fox et al., 2004), and un-

\footnotetext{
* Corresponding author; e-mail: lesleylancaster@abdn.ac.uk
} 
derstanding the genetic basis of these evolutionary transitions may lead to a better predictability of pest outbreaks, and improved mitigation strategies.

A species with a noted propensity for adaptation to naïve hosts is the cowpea seed beetle (Callosobruchus maculatus; Fabricius, 1775) (Wasserman \& Futuyma, 1981; Dick \& Credland, 1984; Messina \& Jones, 2009). These are a common pest species of stored legumes and are extremely detrimental to economic interests and food security throughout the tropical and subtropical world (Boeke et al., 2004). The species originates from West Africa where the cowpea (Vigna unguiculata) is their host legume, but populations of $C$. maculatus have also spread to temperate regions, following human trade, and have adopted several novel agriculturally-cultivated host species associated with these range shifts (Tuda, 2007; Tuda et al., 2014). The beetles exist in small numbers in the field but undergo exponential population growth when the legumes are moved to storage (Messina, 1998). In Nigeria, they can infest an entire stored cowpea harvest within three to five months, corresponding to four to six generations (Singh, 1977). Due to a short generation time and high fecundity, they are responsible for $90 \%$ of all insect damage to stored cowpeas (Caswell, 1981), and they also represent a significant threat to other stored legumes as alternative host races have spread throughout the world (Tuda et al., 2014). Although this makes $C$. maculatus a persistent problem pest species, it also makes them ideally suited for studies investigating the adaptation of species to new native hosts (Tuda, 2007).

Callosobruchus maculatus females deposit their eggs individually on dried beans, with individual females able to lay more than 100 eggs (Messina \& Karren, 2003). After oviposition, larvae emerge from the eggs and burrow into the beans, which they use as a food source as they develop through four larval instars (Messina \& Durham, 2013). They typically consume no food or water once they emerge from the beans as adult beetles. Thus it is the larvae which cause the damage to stored crops. From oviposition to adult emergence is usually 24 days but varies with environmental conditions, population characteristics, and host legume species (Beck \& Blumer, 2014). Adult lifespan is shorter than larval development time, so this species has non-overlapping generations in the laboratory, where individual generations are initiated at fixed time points. The similarity between environmental conditions in crop storage and in the laboratory means that experimental evolution with $C$. maculatus host shifts can be reliably compared to local adaptation in wild populations (Messina, 1998).

C. maculatus has become a model system for using experimental evolution to identify the fitness consequences and genetics associated with host plant shifts in herbivorous insects (Fricke \& Arnqvist, 2007, Gompert \& Messina, 2016). However, previous studies in this system have focused on relatively long-term changes in host use, of at least 10 generations (Messina \& Karren, 2003; Messina, 2004a). In the current study, under no-choice conditions, we evaluated the ability of $C$. maculatus populations to adapt to novel bean types over each of 5 generations fol- lowing exposure to a novel host. This allows us to identify the slope and curvature of the adaptation trajectory during the first few generations on a novel host. We also examined whether the initial stages of adaptation were more likely to involve behavioural changes (changes in host plant acceptance by females, assessed as fecundity) or physiological changes associated with the larva's ability to metabolize the plant material (assessed as offspring survival to adulthood and development time) (Jaenike, 1990). Although fecundity has an underpinning physiological response due to assimilation of nutrients, it is often used as a measure of behavioural adaptation. Fox (1993) found a strong correlation between lifetime fecundity and preference for the most abundant host. We also measured the narrow-sense heritability of fecundity and offspring larval survival across all novel, and the ancestral, bean types.

We hypothesized that if fitness trade-offs generally limit the capacity to adapt to novel hosts in this species, then our population's history of long term, enforced dietary specialization (see 'source population', below) would have eroded additive genetic variation for alternative host use, and the ability to use multiple hosts would have been lost. Alternatively, rapid adaptation to novel hosts following conditions of enforced specialisation would provide evidence for a lack of trade-off between dietary specialisation on ancestral hosts and the ability to adopt new hosts. Furthermore, we predict that an accelerating rate of adaptation to novel hosts (initially slow but gaining momentum) would suggest that substantial genetic changes must occur to release additive genetic variation and expose it to selection. For example, disruption of linkage groups or epistatic networks that confer an advantage under enforced dietary specialization may need to occur before host shifts can effectively be completed. Alternatively, we hypothesized that a decelerating rate of adaptation (very rapid in initial generations but slowing towards an adaptive peak) would indicate that ample additive genetic variation is available, and is unshielded by the ancestral genetic architecture. The latter trend would more strongly indicate that physiological trade-offs between dietary specialisation and adoption of novel hosts are absent in this species, while the former trend would suggest that trade-offs may be present, but genetic variants affected by this trade-off are shielded from directional selection in the specialist condition by regulatory (epistatic) or architectural (linkage) aspects of the species' genetic architecture. Either of these scenarios - lack of trade-offs or shielding genetic architectures - would promote rapid host shifts, and one or both of these may be typical features of emerging pest species' genomes.

\section{MATERIAL AND METHODS}

\section{Source population}

The C. maculatus (Coleoptera: Bruchinae) beetles used were provided by Paul Eady from a population kept at the University of Lincoln, where they were kept on cowpeas (Vigna unguiculata), at a constant temperature of $27^{\circ} \mathrm{C}$ and $35 \%$ relative humidity with a $24 \mathrm{~h}$ light photoperiod in outbred stock populations for at least 19 years or around 285 generations (Eady et al., 2000; P. Eady, pers. comm.). This population was originally obtained from Nia- 
mey, Niger, where they also primarily targeted cowpeas in the wild. For the current study, the beetles were kept at a constant temperature of $27^{\circ} \mathrm{C}$ and uncontrolled humidity with a $24 \mathrm{~h}$ light photoperiod.

\section{Adaptation study}

\section{Establishment of beetle colonies for each line}

To determine the speed of adaptation to a number of novel hosts, we employed experimental evolution via quasi-natural selection. Unlike in artificial selection studies, laboratory natural selection involves exposing populations to controlled, novel environments and leaving them to evolve without any investigator interference into the selected phenotypes or group processes that may be involved in the process of adaptation (Garland \& Rose, 2009; Kawecki et al., 2012). Five new experimental lines were established on each of eight novel host treatments and a control $(n=45$ experimental lines total). The novel and control host treatments were: cowpeas (Vigna unguiculata) (control and ancestral host), mung beans (Vigna radiata), adzuki beans (Vigna angularis), chick peas (Cicer arietinum), lentils (Lens culinaris), soybeans (Glycine max), and 3 cultivated varieties of Phaseolus vulgaris: flageolet beans, haricot beans, and pinto beans. The beetles were passed through one generation on black-eyed beans in their new laboratory at the University of Aberdeen before being divided into experimental lines and transferred to their novel hosts. Upon adult emergence, 100 beetles (50 males and 50 females) were randomly selected and established on $100 \mathrm{~g}$ of fresh beans in $500 \mathrm{ml}$ perspex boxes with mesh lids for ventilation. This design was kept constant across each of the generations.

At each generation, we measured: host acceptance [which comprises both oviposition preferences and fecundity $=$ total eggs laid/number of females (50)], offspring viability (ratio of numbers of emerged beetles to total eggs laid), generation time (here we use a non-standard definition of generation time which tracks changes in age of first recruitment within that cohort; i.e., the time from the date of establishment of each generation to the date of the first emergence of the next generation), population growth rate $(\lambda)$, and per capita rate of increase (r). Population growth was calculated as $\lambda_{\mathrm{i}}=\mathrm{N}_{(\mathrm{t}+1)} / \mathrm{N}_{(\mathrm{t})}$ where $\mathrm{N}_{(\mathrm{t}+1)}$ is final population size at the end of each generation $i$, and $\mathrm{N}_{(\mathrm{t})}$ is the starting population of 100 individuals at the start of each generation. Per capita rate of increase ( $\mathrm{r}$ ) was calculated as $\mathrm{r}_{i}=\operatorname{In}\left[\mathrm{R}_{0}\right] / \mathrm{T}_{i}$ where $\mathrm{R}_{0}$ is the number of offspring produced per female within generation $i$, and $\mathrm{T}_{i}$ is generation time (time in months between initiating the generation with 100 individuals and the emergence date) for each generation. Data were collected for each of five generations for cowpeas, mung beans, adzuki beans, and chickpeas. Data for lentils were collected for two generations and soybeans for three generations then discontinued due to their longer generation time and time constraints on completing the project. No beetles emerged on any variety of Phaseolus vulgaris, so these lines were discontinued.

The common method of estimating C. maculatus beetle numbers is through volume (Messina \& Johnson, 2014) but due to the smaller number of beetles used in this study, a precise number of males and females was preferred in order to prevent changes to effective population sizes $(\mathrm{Ne})$ caused by uncontrolled variation in sex ratio. To reduce their activity and make counting easier, the beetles were chilled in a container submerged in an ice water bath to reduce movements, and randomly selected until 50 males and 50 females had been gathered. It is possible that chilling affected the future fitness of the beetles. To mitigate this effect, the time that beetles were chilled was kept constant between control and treatment lines.

\section{Estimation of fecundity and offspring viability}

After each generation of beetles died they were removed from the container so that their eggs could be counted. This was done by counting the eggs on 100 beans from each replicate line, counting the total beans per container and calculating the total eggs laid from the formula: Total Eggs $=($ eggs counted/bean $) \times($ total beans/container). After emergence, 50 males and 50 females from each line were removed to be placed on a fresh $100 \mathrm{~g}$ of the same bean type to start the next generation. After the remaining beetles from the same generation emerged as adults and had died, their numbers were counted and added to the 100 removed beetles, to obtain a count of total emerged adults. Viability was then estimated from emergence rates $=$ total emerged adults/total eggs laid.

\section{A note on generation times relating to the establishment of new generations}

The 100 individuals used to start the next generation of each line were gathered once approximately 100 individuals had emerged as adults from the previous generation (typically within $48 \mathrm{~h}$ of the emergence of the first adult). This meant that each successive generation was formed from the earliest to emerge, which may have imposed an unwanted artificial selection bias for earlier emergence dates. If the beetles were not collected at this stage but left alone or transferred to a holding container until all had emerged as adults, they would have begun to lay eggs and to age before being transferred to new beans, resulting in the fecundity measurements being inaccurate. This is a source of unwanted artificial selection on generation times rarely mentioned in other studies. As control and treatment lines were treated similarly, differences between novel and control hosts in generation times would be expected to reflect differences in their degree of adaptation to the novel host type.

\section{Heritability study}

To estimate the narrow-sense heritability of maternal characteristics promoting adaptation to novel hosts (fecundity and offspring viability), unrelated virgin females ( $f_{0}$ ) were mated in sets of three females to one male in a half-sib mating design (Lynch $\&$ Walsh, 1998). One $f_{1}$ female offspring from each of these $f_{0}$ matings was again randomly mated to a male unrelated to any other individuals used in the heritability study, and data on her fecundity (total number of eggs laid) and her offspring viability were obtained. Our $\mathrm{F}_{0}$ males and females were obtained from each of four bean types at the end of generation 1 in the adaptation study (cowpeas, chickpeas, adzuki beans, and mung beans), mated within bean types, and provided the same bean type as oviposition substrate ( $5 \mathrm{~g}$ beans provided to each female). Thus the 60 half-sibs analysed were nested within 5 half-sib families per bean type (see analysis, below). This approach generates heritability estimates that are characteristic of the base population, but ensures equal representation of genotypes which can survive on alternative bean types, and also accounts for variation in environmental effects that may arise from different host types (i.e., we assessed heritability of fitness components of the base population under conditions of a variable host environment).

\section{Statistical analysis \\ Novel hosts}

We examined the slopes and shapes of adaptation trajectories using mixed models in the packages lme4 and lmerTest (Bates et al., 2014; Kuznetsova et al., 2016) for R (ver. 3.1.3) (R Core Team, 2013). Measurements for fecundity and egg/larval viability and calculated values of $\lambda$ and $r$ for each replicate in each generation were analysed as response variables with fixed factors for bean type (cowpea as the baseline), generation (1-5), generation ${ }^{2}$ 
and all interactions. A random effect was included to account for individual variation among replicates. For each model, we fitted a Gaussian error structure and tested the residuals for conformity to the assumptions of this error structure. For each response variable, we estimated the full model and all reduced models, and selected the best-fit model using AICc, implemented in the AICcmodavg package for R (Anderson et al., 2000; Mazerolle, 2016). To further address our hypothesis that changes in the rate of adaptation over time exhibit significant curvature (accelerating or decelerating rates of change in adaptation to novel hosts with respect to controls), we also examined the direction and significance of the effect of: bean type $\times$ generation $^{2}$, using type III sums of squares in the car package for R (Fox \& Weisberg, 2011).

\section{Heritability}

Phenotypic variance in fecundity and offspring viability from the $f_{1}$ females in the heritability experiment were partitioned into paternal and host species sources of variance by fitting random, nested effects in the lme4 package for R (Bates et al., 2014), with sires nested within bean type according to the following partitioning of phenotypic variance: $\sigma_{\mathrm{z}}^{2}=\sigma_{\mathrm{s}(\mathrm{b})}^{2}+\sigma_{\mathrm{e}}^{2}$, where $\sigma^{2}$ represents the total phenotypic variance, $\sigma_{s(b)}^{2}$ represents variance due to sires, nested within bean type, and $\sigma^{2}$ represents the residual variance due to females and environmental variance (Lynch \& Walsh, 1998). A separate model was used for each of the fecundity and viability response variables. Because we offered individual females relatively few beans, and under these circumstances fecundity and viability can be correlated with each other (if increased fecundity reduces larval survival via competition effects), we regressed emergence rates (viability) on fecundity prior to analysis. Thus we calculated the heritability of residual emergence rates, to generate an estimate of genetic variation for viability under (typical) conditions of larval crowding, but which is independent of heritability of fecundity. We then calculated heritability using the formula $h^{2}=4\left(\sigma_{s(b)}^{2}\right) / \sigma_{z}^{2}$, where $\sigma_{s(b)}^{2}$ is the phenotypic variance attributable to sire, and $\sigma^{2}$ is the total phenotypic variance in the trait (Lynch \& Walsh, 1998). To estimate uncertainty in the heritability of each trait, $95 \%$ confidence intervals of the variance components were estimated, and heritability recalculated using the confidence limits instead of the mean.

\section{RESULTS}

Adaptation to novel bean types was initially rapid, but declined over time as fitness values approached ancestral optima (Table 1, Fig. 1). Specifically, evidence of adaptation over time was observed on chickpeas and lentils, whereas evidence for mildly-accumulating maladaptation was observed for adzuki beans, mung beans, and soya beans (summarised in Table 1, and described in more detail in the following paragraphs). Overall, adaptive changes outweighed maladaptive changes in magnitude, resulting

Table 1. Signatures of adaptation on each bean type, and evidence for non-linear rates of adaptation for each component of fitness (+ indicates adaptation over time, - indicates declining fitness over time, 0 indicates no evidence for change in fitness over time).

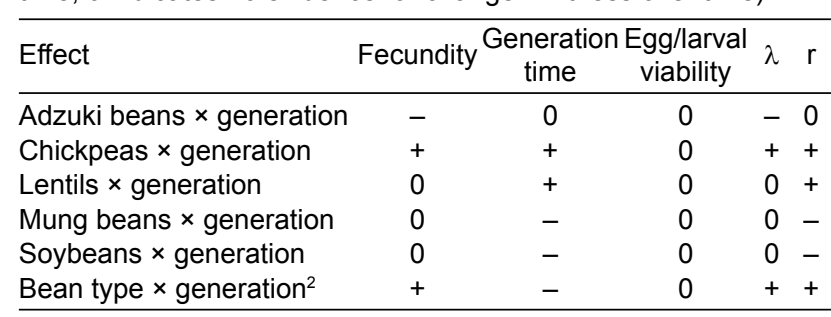

in significant evidence of adaptation across bean types, which was initially rapid but declined over time at the approach of the ancestral fitness values (final row of Table 1, Fig. 1). Best fit models for specific components and measures of fitness are described below.

\section{Beetle acceptance to novel host (fecundity)}

The best fit model for fecundity included the fixed effects: bean type, generation, bean type $\times$ generation, and generation $^{2}$ (Table 2). Fecundity was significantly lower on chickpeas and soybeans compared to control lines, and fecundity improved over time in chick peas, in comparison to control lines. In contrast, fecundity declined slightly over time in adzuki beans, suggesting ongoing maladaptation and loss of genetic variation for fitness on the new host. For the full model, the effect of bean type $\times$ generation $^{2}$ was significant using type III sums-of-squares $\left(\chi^{2}=17.21\right.$, $P=0.002$ ), indicating that the rate of adaptation to new hosts declined over time, at the approach of a phenotypic optimum (which differed among host types; Fig. 1A). We also ran a separate model limited to the first generation, to better represent the bean types on which emergence of generation 1 eggs was unsuccessful. The results of this model confirmed that there was no significant difference between fecundity on haricot/pinto/flageolet beans (Phaseolus vulgaris), compared to the fecundity of control beetles in generation 1 ( $P=0.77,0.24$, and 0.72 , respectively). Per-bean fecundity was highest overall on cowpeas (average of 6.1 eggs laid per bean), followed by adzuki beans/chickpeas (5.4 eggs/bean), soybeans (1.35 eggs/bean), mung beans (2.2 eggs/bean), and lentils ( 0.7 eggs/bean).

\section{Generation time}

The best fit model explaining generation time included the fixed effects: bean type, generation, bean type $\times$ generation, and generation ${ }^{2}$ (Table 3 ). Generation times were significantly longer on adzuki beans, chickpeas, lentils and soybeans, in comparison to generation times in control lines, and only mung beans resulted in unchanged generation times in comparison to controls. Adaptation to novel hosts in generation time was observed for chickpeas and lentils (significant, negative bean type $\times$ generation effect,

Table 2. Fixed effects in the best-fit model explaining fecundity.

\begin{tabular}{|c|c|c|c|c|c|}
\hline Fixed effects & Estimate & SE & df & $\mathrm{t}$ value & $\operatorname{Pr}(>|t|)$ \\
\hline Intercept & 26.70 & 5.19 & 124 & 5.147 & $<0.001^{* * *}$ \\
\hline Bean type: Adzuki & 11.17 & 5.49 & 124 & 2.034 & $0.04^{*}$ \\
\hline Bean type: Chickpeas & -25.99 & 5.49 & 124 & -4.734 & $<0.001^{* * *}$ \\
\hline Bean type: Flageolet & -2.03 & 4.78 & 124 & -0.424 & 0.67 \\
\hline Bean type: Haricot & -1.75 & 4.78 & 124 & -0.366 & 0.72 \\
\hline Bean type: Lentil & -8.51 & 9.47 & 124 & -0.899 & 0.37 \\
\hline Bean type: Mung & 7.70 & 5.49 & 124 & 1.402 & 0.16 \\
\hline Bean type: Pinto & -5.46 & 4.78 & 124 & -1.141 & 0.26 \\
\hline Bean type: Soybean & -49.70 & 7.09 & 124 & -7.010 & $<0.001^{* * *}$ \\
\hline Generation & 19.51 & 3.17 & 124 & 6.148 & $<0.001^{* * *}$ \\
\hline Generation $^{2}$ & -2.63 & 0.49 & 124 & -5.346 & $<0.001^{* * *}$ \\
\hline Adzuki $\times$ Generation & -3.84 & 1.66 & 124 & -2.320 & $0.02^{*}$ \\
\hline Chickpea $\times$ Generation & 3.17 & 1.66 & 124 & 1.916 & 0.06 \\
\hline Lentil $\times$ Generation & 2.33 & 5.56 & 124 & 0.418 & 0.68 \\
\hline Mung $\times$ Generation & 0.35 & 1.66 & 124 & 0.214 & 0.83 \\
\hline Soybean $\times$ Generation & 4.74 & 3.03 & 124 & 1.565 & 0.12 \\
\hline
\end{tabular}



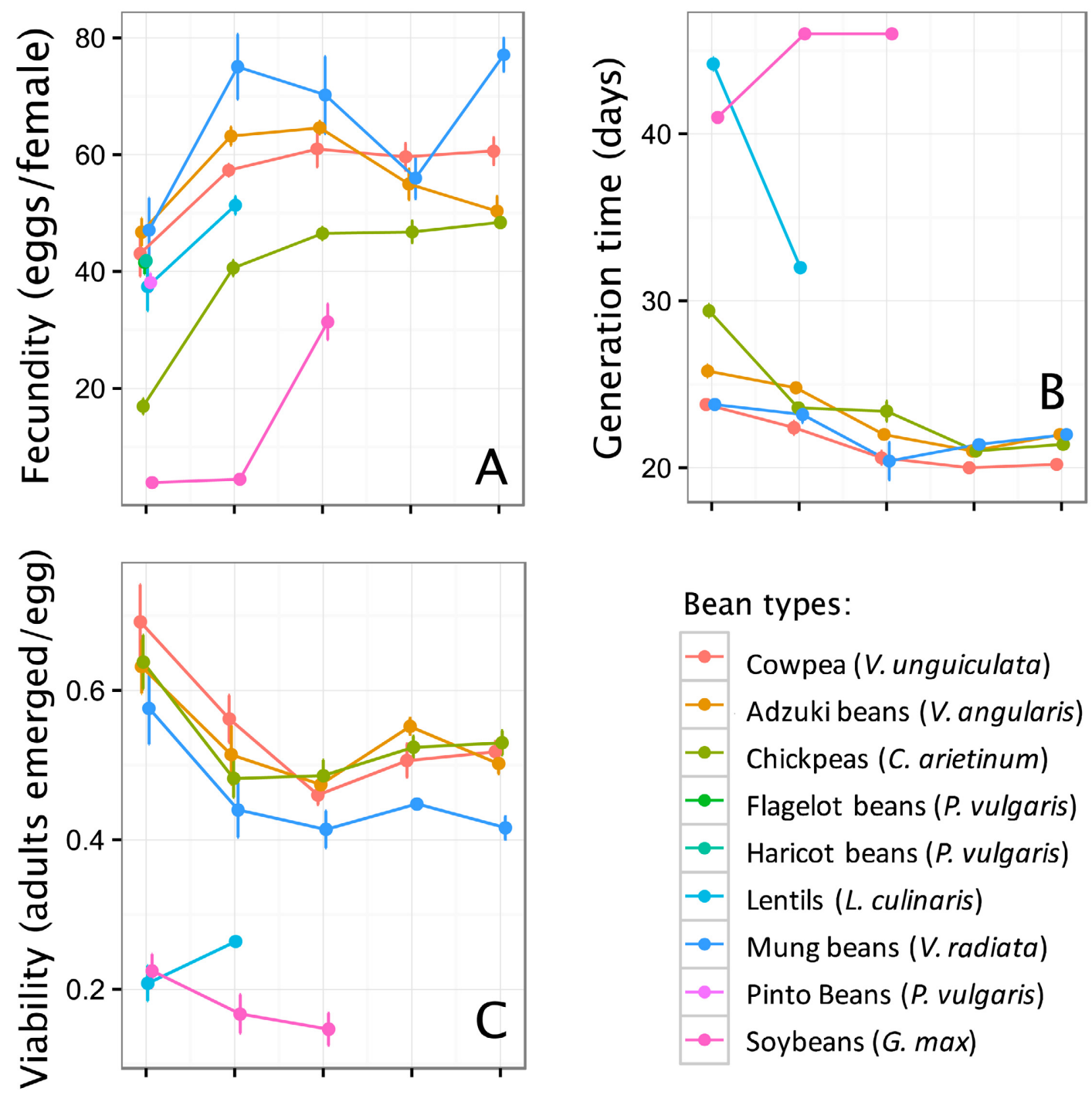

\section{Bean types:}
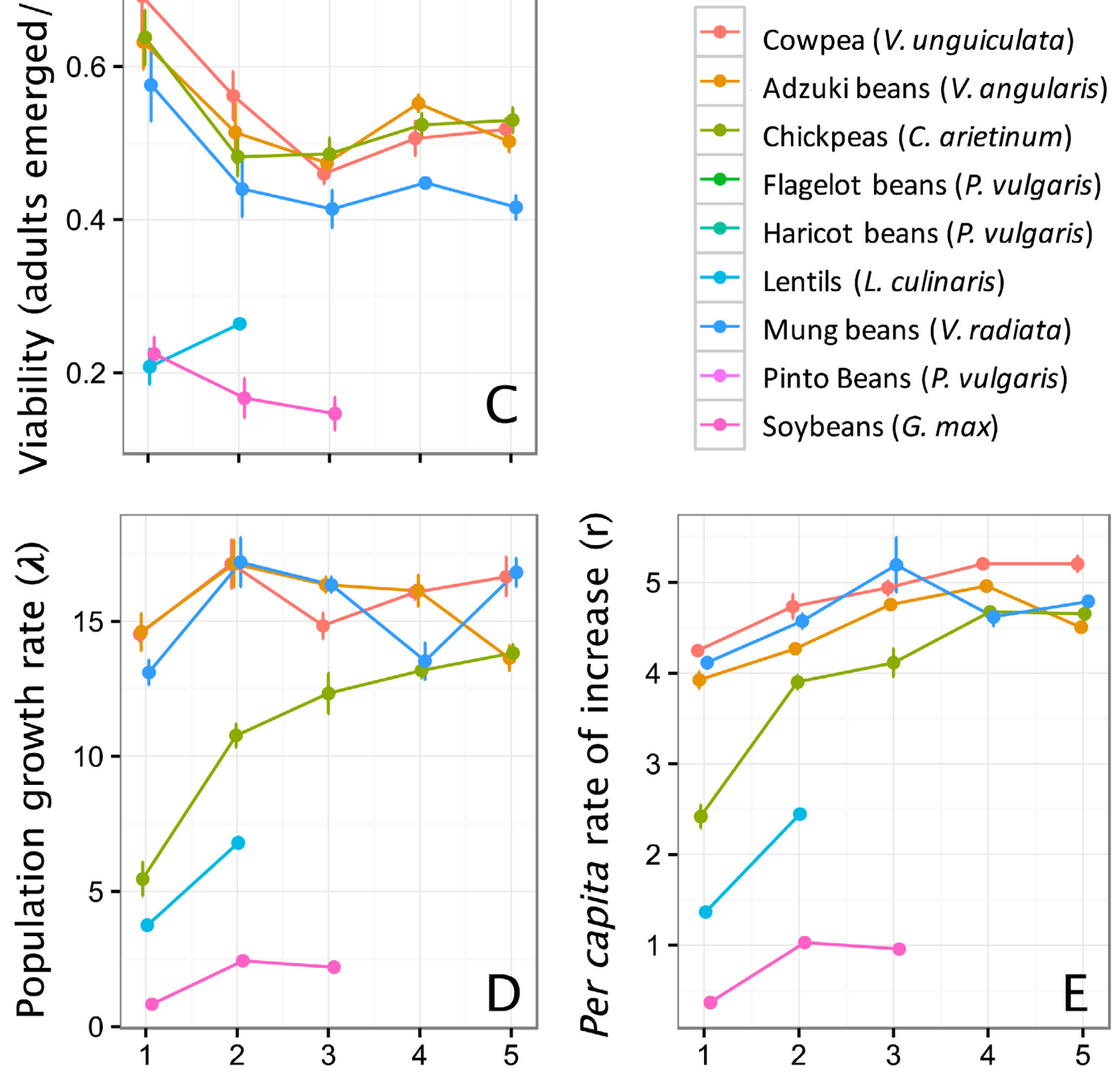

\section{Generations of adaptation to novel host}

Fig. 1. Changes in fitness-related traits and population fitness over 5 generations of experimental evolution in the laboratory (cowpea lines represent the ancestral controls). Each line represents the mean and standard errors for 5 replicates per bean type. A) Fecundity, B) Generation time, C) Offspring viability, D) Population growth rate, and E) per capita rate of population increase. Rapid adaptation can be observed within the first few generations of adaptation to novel hosts, despite a long evolutionary history of dietary specialisation in this laboratory population. 
Table 3. Fixed effects in the best-fit model explaining generation time (in days).

\begin{tabular}{|c|c|c|c|c|c|}
\hline Fixed effects & Estimate & SE & df & $\mathrm{t}$ value & $\operatorname{Pr}(>|t|)$ \\
\hline (Intercept) & 27.36 & 0.67 & 112 & 40.713 & $<0.001^{* * *}$ \\
\hline Bean type: Adzuki & 2.20 & 0.71 & 112 & 3.178 & 0.002 ** \\
\hline Sean type: Chickpeas & 5.06 & 0.71 & 112 & 116 & $<0.001^{* * *}$ \\
\hline Bean type: Lentil & 29.92 & 1.23 & 112 & & $<0.001^{* * *}$ \\
\hline Bean type: Mung & -0.50 & 0.71 & 112 & -0.703 & 0.48 \\
\hline ype: Soybean & 13.44 & 0.92 & 112 & 32 & $01^{* * *}$ \\
\hline & & 0.41 & 112 & & $<0.0$ \\
\hline Generation $^{2}$ & 0.44 & 0.06 & 112 & 6.909 & $<0.001^{* * *}$ \\
\hline Adzuki × Generation & -0.18 & 0.21 & 112 & -0.840 & 0.40 \\
\hline Chickpeas $\times$ Generation & -0.90 & 0.21 & 112 & -4.198 & $<0.001^{* * *}$ \\
\hline Lentil $\times$ Generation & -9.92 & 0.72 & 112 & -13.767 & $<0.001^{* * *}$ \\
\hline Mung $\times$ Generation & 0.42 & 0.21 & 112 & 1.959 & 0.05 \\
\hline Soybean $\times$ Generation & 4.34 & 0.39 & 112 & 11.054 & $<0.001^{* * *}$ \\
\hline
\end{tabular}

Table 2), but performance on soybeans and mung beans relating to generation time worsened over time with respect to control lines (significant or marginally significant, positive bean type $\times$ generation effect, Table 3 ). The significant, non-linear decrease in generation time observed across all control and treatment lines likely reflects inadvertent artificial selection on generation time applied to all lines, as described in the methods section. In the full model, the effect of bean type $\times$ generation ${ }^{2}$ was also significant $\left(\chi^{2}=41.60\right.$, $P<0.0001$ ), but this primarily reflected ongoing maladaptation in soya beans, rather than non-linearity in rates of adaptation (Fig. 1B).

\section{Offspring viability}

The best fit model for offspring viability (number of emerged, adult beetles/egg laid) included the fixed effects: bean type, generation, and generation ${ }^{2}$ (Table 4). Offspring viability was significantly lower on lentils, mung beans, and soybeans than controls (Fig. 1C), with no statistical evidence of adaptation of this trait to any of the novel hosts. The type III sums-of-squares ANOVA also indicated an overall insignificant effect of bean type $\times$ generation $^{2}$ in the full model $\left(\chi^{2}=1.96, P=0.74\right)$.

\section{Population growth rate $(\lambda)$}

The best fit model for population growth rate included the fixed effects: bean type, generation, bean type $\times$ generation, and generation ${ }^{2}$ (Table 5). Lentils, soybeans, and chickpeas all had lower population growth rates than controls, with evidence that adaptive changes in population growth rate occurred on chickpeas, while declining population growth rates over time were observed on adzuki beans. In the full model, the effect of bean type $\times$ generation $^{2}$ was

Table 4. Fixed effects in the best-fit model explaining offspring viability (adult offspring per egg).

\begin{tabular}{lrcccc}
\hline Fixed effects & Estimate & SE & df & t value & $\operatorname{Pr}(>|t|)$ \\
\hline Intercept & 0.749984 & 0.029836 & 117 & 25.137 & $<2 \mathrm{e}-16^{\star * *}$ \\
Bean type: Adzuki & -0.012800 & 0.018412 & 117 & -0.695 & 0.49 \\
Bean type: Chickpeas & -0.015600 & 0.018412 & 117 & -0.847 & 0.40 \\
Bean type: Lentil & -0.358180 & 0.025284 & 117 & -14.166 & $<2 \mathrm{e}-16^{\star * *}$ \\
Bean type: Mung & -0.088800 & 0.018412 & 117 & -4.823 & $4.30 \mathrm{e}-06^{\star * *}$ \\
Bean type: Soybean & -0.387184 & 0.021785 & 117 & -17.773 & $<2 \mathrm{e}-16^{\star * *}$ \\
Generation & -0.134210 & 0.021133 & 117 & -6.351 & $4.24 \mathrm{e}-09^{* * *}$ \\
Generation $^{2}$ & 0.018204 & 0.003519 & 117 & 5.174 & $9.61 \mathrm{e}-07^{* * *}$ \\
\hline
\end{tabular}

Table 5. Fixed effects in the best-fit model explaining population growth rates.

\begin{tabular}{lrcccc}
\hline Fixed effects & Estimate & SE & df & t value & $\operatorname{Pr}(>|\mathrm{t}|)$ \\
\hline Intercept & 11.89413 & 0.94628 & 112 & 12.569 & $<0.001^{* * *}$ \\
Bean type: Adzuki & 1.57220 & 1.00134 & 112 & 1.570 & 0.12 \\
Bean type: Chickpeas & -9.51280 & 1.00134 & 112 & -9.500 & $<0.001^{* * *}$ \\
Bean type: Lentil & -12.04244 & 1.72662 & 112 & -6.975 & $<0.001^{* * *}$ \\
Bean type: Mung & -0.60940 & 1.00134 & 112 & -0.609 & 0.54 \\
Bean type: Soybean & -12.86531 & 1.29341 & 112 & -9.947 & $<0.001^{* * *}$ \\
Generation & 2.88192 & 0.57890 & 112 & 4.978 & $<0.001^{* * *}$ \\
Generation & -0.42715 & 0.08968 & 112 & -4.763 & $<0.001^{* * *}$ \\
Adzuki $\times$ Generation & -0.61420 & 0.30191 & 112 & -2.034 & $0.044^{*}$ \\
Chickpeas $\times$ Generation & 1.59480 & 0.30191 & 112 & 5.282 & $<0.001^{* * *}$ \\
Lentil $\times$ Generation & 1.44554 & 1.01464 & 112 & 1.425 & 0.16 \\
Mung $\times$ Generation & 0.05420 & 0.30191 & 112 & 0.180 & 0.86 \\
Soybean $\times$ Generation & -0.48931 & 0.55284 & 112 & -0.885 & 0.38 \\
\hline
\end{tabular}

significant $\left(\chi^{2}=11.15, P=0.02\right)$, indicating a decreasing rate of adaptation at the approach of the ancestral fitness optimum (particularly in chickpeas; Fig. 1D).

\section{Per capita rate of population increase $(r)$}

The best model explaining per capita rates of increase included terms for bean type, generation, bean type $\times$ generation and generation ${ }^{2}$ (Table 6). Chickpeas, lentils, and soybeans all showed significantly lower per capita rate of population increase than the control, but per capita increases improved over time in chickpeas and lentils. Soybeans and (marginally) mung beans showed a decline in per capita population rate over the generations (Table 6). In the full model, the effect of bean type $\times$ generation $^{2}$ was significant $\left(\chi^{2}=12.20, P=0.02\right)$, further indicating slowing rates of adaptation at the approach of a phenotypic optimum (Fig. $1 \mathrm{E})$.

\section{Heritability study}

The second objective of this study was to explore the heritability of two fitness components, fecundity and offspring viability. For fecundity, estimated $h^{2}=1.03 \pm 0.43$ (95\% CI). For residual offspring viability under (typical) crowded conditions, estimated $h^{2}=0.38 \pm 0.16$ (95\% CI).

\section{DISCUSSION}

Our experimental lines of $C$. maculatus were able to quickly adapt to 2 out of the 5 novel host species (chick peas and lentils), and the rapid and dramatic adaptation ob-

Table 6. Fixed effects in the best-fit model explaining per capita rate of population increase.

\begin{tabular}{|c|c|c|c|c|c|}
\hline Fixed effects & Estimate & SE & df & $\mathrm{t}$ value & $\operatorname{Pr}(>|t|)$ \\
\hline Intercept & .22826 & 0.15860 & 112 & 20.355 & $<0.0$ \\
\hline Bean type: Adzuki & & .16783 & 112 & & \\
\hline ean type: Chickpeas & -1.7 & 0.16783 & 112 & & \\
\hline ean type: Lentil & -3.2 & 0.28939 & 112 & & \\
\hline & & 783 & & & \\
\hline & -3.4 & 0.21678 & 112 & & $<0$ \\
\hline & & 9702 & 112 & & \\
\hline & & & 112 & & \\
\hline & -0. & 0.05060 & 112 & & \\
\hline & 0.28627 & 0.05060 & 112 & & $<0$ \\
\hline & & 0.17006 & 112 & & $<0$ \\
\hline & -0.09873 & 0.05060 & 112 & & \\
\hline Soybean $\times$ Generation & -0.20765 & 0.09266 & 112 & -2.241 & 0.03 * \\
\hline
\end{tabular}


served in these two host species resulted in an overall positive and significant signature of adaptation across novel hosts (Table 1). Gains in fitness were most dramatic in the first several generations of selection, after which fitness gains declined as the population approached its former adaptive optimum (Fig. 1D, E, Orr, 2005), reflected in a significant interaction of bean type with generation ${ }^{2}$ for the major indices of population fitness ( $\lambda$ and $r$; Table 1 and results section). This result supported our hypothesis that the population possesses ample standing additive genetic variation for host plant shifts, despite nearly 300 generations of enforced specialization in the lab (and an even longer-term evolutionary history of predominantly targeting cowpeas in the ancestral Nigerian source population), and despite the fact that phenotypic plasticity and GxE effects may have in part contributed to rapid adaptation. This result indicates that trade-offs do not strongly limit the ability of $C$. maculatus to adapt to a novel host, at least during a single episode of selection (see Gompert \& Messina, 2016 for an instance where trade-offs emerged under fluctuating selection), and thus genetic architecture (for example, to shield adaptive genetic variants in the new host from trade-offs with fitness on the ancestral host) likely does not play a strong role in limiting the rate of adaptation to novel hosts.

Adaptation to novel hosts was behavioural as well as physiological - where fitness gains occurred, these reflected both changes in oviposition behavior as well as changes in generation time (Table 1). For adaptation to lentils, fitness gains were entirely reflected in changes in generation time, whereas adaptation to chickpeas reflected adaptive changes in both oviposition behavior and generation time. In no case did fitness gains reflect changes in offspring survival, indicating that traits contributing directly to offspring viability may be much less evolvable than behavioural or phenological traits (despite high heritability of offspring viability, see below). This result concurs with past studies, which have generally found that host acceptance evolves faster than related physiological changes needed to increase offspring viability (Futuyma et al., 1984; Messina, 2004b).

For increased larval survival or increased development times, a physiological change is required (Jaenike, 1990). Previous studies have produced conflicting results regarding whether physiological changes will arise in less than 40 generations. For example, Wasserman \& Futuyma (1981) found no physiological adaptations, such as increasing digestive efficiency over time, when $C$. maculatus were switched to a new host for 11 generations. They observed only behavioural changes associated with adaptation over this time period. Conversely, Fricke \& Arnqvist (2007) did find physiological evolutionary changes within 35 generations of selection, associated with an accelerated growth rate on novel hosts. Although the current study was carried out for only five generations, faster development times were observed on the novel hosts over time with respect to controls (cowpeas), indicating very rapidly evolving changes in physiological traits associated with developmental time, despite no evidence for evolutionary change in offspring viability over this short time period. However, given that only 2 generations were run on the poorest hosts (lentils, soybeans), it is possible that changes in viability may have been observed in these hosts if run for additional generations. It is noteworthy that, although viability on novel hosts did not change with respect to controls, viability decreased with generation time across all host types (Fig. 1C). This may represent a trade-off between development time and viability (see further discussion of our unwanted artificial selection on development time, below).

Similarly, where maladaptive changes accumulated over time - in adzuki beans, mung beans, and soybeans, these also reflected disadvantageous changes in fecundity or generation time, and never reflected declines in offspring adult emergence rates. Where such fitness declines occurred, they were typically modest in comparison to fitness gains on beans where adaptation occurred (Fig. 1). Declines in fitness may reflect inbreeding and drift where selection was strong and had potentially strong effects on effective population sizes (e.g., on soybeans), or may simply reflect reduced fitness on a marginally sub-optimal resource (e.g., for mung and adzuki beans, where weak selection against most genotypes could result in consistently sub-optimal performance and a failure to adapt overall). The fact that both adaptation and maladaptation to novel resources involved phenological and behavioural traits, rather than physiological competence, suggests that these traits should by examined more closely during efforts to understand and mitigate the spread of crop pests to new hosts in the wild.

This study cannot determine why $C$. maculatus was able to adapt to some bean types but not others. The completely unsuccessful hosts (all $P$. vulgaris varieties) resulted in comparable fecundity to the control lines in the first generation of the study, suggesting that fecundity was not limiting the rate of adaptation to this inhospitable host species. With these completely unsuccessful hosts, egg hatching did occur (pers. observ.) but larvae died during development, likely due to toxins within the seed which kill the larvae when consumed (Janzen, 1977). If these toxins can be overcome, then adaptation to these hosts is a possibility. A recent experiment indicated that this was achievable, with small number emerging from haricot beans in a choice experiment (Leonard \& Lancaster, unpubl. data). However, gains in viability in response to highly indigestible or toxic hosts is likely overall a slower evolutionary process than behavioural or phenological changes required to adapt to suboptimal, but not completely inhospitable, hosts. More work is needed to understand the underpinning genetic architecture of viability adaptations.

Similarly, although we have evidence for rapid adaptation and high heritability for behavioural and phenological traits, we still lack resolution on underpinning causes of these changes. Past studies have shown that rough surfaced seeds of chickpeas provide weaker surfaces for egg laying, leading to reduced fecundity on this bean type (Nwanze et al., 1975; Nwanze \& Horber, 1976). It apparently takes several generations for females to recognize and utilize this novel resource. Similarly, the small size of lentils may ini- 
tially render them unrecognizable or unpreferred (females typically prefer to space eggs evenly across the laying surface, however African populations of $C$. maculatus from which the beetles in this study were derived do not attempt to evenly spread their eggs to the same extent as Asian populations which are also frequently studied; Messina \& Mitchell, 1989). Lentils, chickpeas, and soybeans were the bean types on which fitness was most severely impacted in the first several generations, and these host species are only distantly related to cowpeas (Fig. 1). Mung and adzuki beans are in the same genus as the ancestral host (Vigna), and it is likely this close relationship that mediated the very modest changes in fitness when $C$. maculatus was introduced to these novel hosts (Futuyma, 2000; Messina \& Jones, 2009). This implies switching to hosts within the Vigna require less adaptation than to another genus altogether.

When choosing the point at which beetles would be collected to begin the next generation, we identified a tradeoff between losing fecundity data and adding artificial selection for early emergence times. It was decided to accept the impact of artificial selection, as it was assumed this would affect all populations equally, whereas later collection might make fecundity data erroneous. This source of error was further minimised by using the control as a baseline comparator to the novel hosts; this ensured that inadvertent artificial selection on development time would be included in this baseline and not confound the main results. However, there are other possible implications of this decision. Moller et al. (1990) found a negative correlation between development time and fecundity, which may be responsible for the decrease in the number of eggs laid in later generations, seen most especially on the adzuki beans, as the effects of the artificial selection for the development rate became stronger with each generation.

The variation among host species in egg density per bean (see results) was largely correlated with bean size: chick peas are the largest, and they generally received high numbers of eggs per bean (5.4 eggs/bean), lentils the smallest with the lowest numbers of eggs per bean $(0.7$ eggs/ bean). Nonetheless variation in larval competition within beans (number of larvae per unit of bean mass) could act to reduce among-host variation in offspring viability and fecundity in later generations, via effects on female body size (e.g., if fecundity becomes too high on a favourable host, larval viability may decrease, and smaller body sizes may slightly reduce fecundities in the next generation. Such density dependent effects may limit adaptation beyond levels observed in the control lines in our experimental populations. Fluctuations in fecundity and viability in the baseline (control) lineages likely in part result in this competition-based trade-off.

We estimated narrow-sense heritability for fecundity and larval viability. If low heritability is found, which is often the case with fitness-related traits because selection has already eroded much of the additive genetic variation for the trait, this implies that the trait would not respond quickly to environmental changes (Freeman \& Heron, 2014). In this heritability study, both fecundity and offspring viability were found to have high heritability. In both cases, these results appeared relatively high in comparison to typical heritability calculations, but they are not without precedent within herbivorous insects (Mousseau \& Roff, 1987). Previously, using a half-sib design, heritability of egg length in C. maculatus was calculated as $0.74 \pm 0.17$ (Fox, 1994) and oviposition preference had a heritability of 0.73 (Fox, 1993). In the current study, we estimated heritability as $1.03 \pm 0.43$ for fecundity and $0.75 \pm 0.32$ for offspring viability, suggesting that genetic factors predominate over environmental ones, and to date, this applies to all four traits investigated both in this study and in earlier studies by Fox (1993, 1994). More work is needed to determine why $C$. maculatus, and potentially other pest species, retain such high genetic capacity to rapidly adapt to novel hosts, even after long-term dietary specialisation. Further studies are also required to understand why high heritability of fecundity translated into rapid evolutionary change, but that offspring viability consistently failed to evolve, despite such high heritability for this trait.

The results of this study provide novel evidence that host shifts in pest species can proceed via extremely rapid adaptation, indeed with most fitness gains occurring within the first 5 generations of exposure to a novel host species. Such rapid adaptation following even the first and second generations of selection suggests that extreme caution must be used when inferring environmental parental effects on host use traits, because parents' rearing environment is also in fact often a strongly selective environment. Thus parents, originally from a shared gene pool but reared in alternative environments, likely reflect differing derived gene pools, after even a single generation of selection. (See also Fox et al., 1995: "The symmetry between the maternal and paternal host effects on survivorship suggests that they represent a response to intense selection during the experiment, and suggests the potential for rapid adaptation to this host" based on their work on parental effects in the seed beetle Stator limbatus.) Further understanding the basis of very high levels of standing additive genetic variation for fitness on novel hosts in pest species, even after prolonged periods of enforced specialization, is the subject of our ongoing work. Similarly, understanding why this genetic variation does not always correspond to evolutionary change (here, in the case of a failure of larval viability to evolve), also requires further investigation. It was previously appreciated that $C$. maculatus adapts rapidly to novel hosts within 10-20 generations (Messina, 1993; Messina \& Durham, 2015), but until this study, it was little appreciated how incredibly rapid these evolutionary changes can be.

ACKNOWLEDGEMENTS. Thank you to P. Eady for providing C. maculatus to initiate our laboratory population and advice on rearing them. The study was funded by the University of Aberdeen core teaching funds (honours project budget to TP), and by a doctoral training grant to AL from the BBSRC-EastBio doctoral training partnership. 


\section{REFERENCES}

Anderson D.R., Burnham K.P. \& Thompson W.L. 2000: Null hypothesis testing: Problems, prevalence, and an alternative. $-J$. Wildl. Manag. 64: 912-923.

Bates D., Mächler M., Bolker B. \& Walker S. 2014: Fitting linear mixed-effects models using lme4. - J. Stat. Softw. 67: 51.

Bebber D.P., Ramotowski M.T. \& GuRR S.J. 2013: Crop pests and pathogens move polewards in a warming world. - Nat. Clim. Chang. 3: 985-988.

Beck C.W. \& Blumer L.S. 2014: A Handbook on Bean Beetles, Callosobruchus maculatus. National Science Foundation. URL: http://www.beanbeetles.org/handbook.pdf (last accessed 16 June 2015).

Boeke S.J., Van Loon J.J.A., Van Huis A. \& Dicke M. 2004: Host preference of Callosobruchus maculatus: A comparison of life history characteristics for three strains of beetles on two varieties of cowpea. - J. Appl. Entomol. 128: 390-396.

Bowers M.D., Stamp N.E. \& Collinge S.K. 1992: Early stage of host range expansion by a specialist herbivore, Euphydryas phaeton (Nymphalidae). - Ecology 73: 526-536.

CAswell G.H. 1981: Damage to stored cowpea in the northern part of Nigeria. - Samaru J. Agric. Res. 1: 11-19.

Dick K.M. \& CREdLAND P.F. 1984: Egg production and development of three strains of Callosobruchus maculatus (F.) (Coleoptera: Bruchidae). - J. Stor. Prod. Res. 20: 221-227.

EAdY P.E., WILSON N. \& JACKSON M. 2000: Copulating with multiple mates enhances female fecundity but not egg-to-adult survival in the bruchid beetle Callosobruchus maculatus. Evolution 54: 2161-2165.

Fox C.W. 1993: A quantitative genetic analysis of oviposition preference. - Evolution 47: 166-175.

Fox C.W. 1994: Maternal and genetic influences on egg size and larval performance in a seed beetle (Callosobruchus maculatus): multigenerational transmission of a maternal effect? Heredity 73: 509-517.

Fox J. \& Weisberg S. 2011: Multivariate Linear Models in R. An $R$ Companion to Applied Regression. 2nd ed. Sage. URL: https://cran.r-project.org/web/packages/car/car.pdf

Fox C.W., Waddell K.J. \& Mousseau T.A. 1995: Parental host plant affects offspring life histories in a seed beetle. - Ecology 76: $402-411$.

Fox C.W., Stillwell R.C., Amarillo A.R., Czesak M.E. \& MessiNA F.J. 2004: Genetic architecture of population differences in oviposition behaviour of the seed beetle Callosobruchus maculatus. - J. Evol. Biol. 17: 1141-1151.

FreEman S. \& Herron J.C. 2014: Evolution at multiple loci: Quantitative genetics. In Freeman S. \& Herron J.C. (eds): Evolutionary Analysis. Pearson Education, Harlow, Essex, pp. 335-376.

Fricke C. \& ARnQvist G. 2007: Rapid adaptation to a novel host in a seed beetle (Callosobruchus maculatus): The role of sexual selection. - Evolution 61: 440-454.

Futuyma D.J. 2000: Potential evolution of host range in herbivorous insects. In Driesche R.V., Heard T., McClay A. \& Reardon R. (eds): Proceedings Host-Specificity Testing of Exotic Arthropod Biological Control Agents: The Biological Basis for Improvement in Safety, Bozeman, Montana, USA, July 4-14, 1999. USDA Forest Service, Morgantown, West Virginia, pp. 42-53.

FutuYMa D.J., CORT R.P. \& VAN NoORDWIJK I. 1984: Adaptation to host plants in the fall cankerworm (Alsophila pometaria) and its bearing on the evolution of host affiliation in phytophagous insects. - Am. Nat. 123: 287-296.
Garland T., Rose M.R. \& Michael R. 2009: Experimental Evolution: Concepts, Methods, and Applications of Selection Experiments. University of California Press, Berkeley, Los Angeles, $730 \mathrm{pp}$.

GOMPERT Z. \& Messina F.J. 2016: Genomic evidence that resource-based trade-offs limit host-range expansion in a seed beetle. - Evolution 70: 1249-1264.

Gutierrez D. \& Thomas C.D. 2000: Marginal range expansion in a host-limited butterfly species Gonepteryx rhamni. - Ecol. Entomol. 25: 165-170.

JAENIKE J. 1990: Host specialization in phytophagous insects. Annu. Rev. Ecol. Syst. 21: 243-273.

JANZEN D.H. 1977: How southern cowpea weevil larvae (Bruchidae: Callosobruchus maculatus) die on nonhost seeds. - Ecology 58: 921-927.

Joshi A. \& THOMPSON J.N. 1995: Trade-offs and the evolution of host specialization. - Evol. Ecol. 9: 82-92.

Kawecki T.J., Lenski R.E., Ebert D., Hollis B., Olivieri I. \& Whiтlock M.C. 2012: Experimental evolution. — Trends Ecol. Evol. 27: 547-560.

Koizumi T., Fusiyama N. \& Katakura H. 1999: Host-plant specificity limits the geographic distribution of thistle feeding ladybird beetles. - Entomol. Exp. Appl. 93: 165-171.

Kuznetsova A., Brockhoff P.B. \& Christensen R.H.B. 2016: Tests in Linear Mixed Effects Models Version. Cran. URL https://cran.r-project.org/web/packages/lmerTest/lmerTest.pdf

LANCASTER L.T. 2016: Widespread range expansions shape latitudinal variation in insect thermal limits. - Nat. Clim. Chang. 6: 618-621.

LYNCH M. \& WALSH B. 1998: Sib analysis. In Lynch M. \& Walsh B. (eds): Genetics and Analysis of Quantitative Traits. Sinauer Associates, Sunderland, MA, pp. 553-579.

Mazerolle M.J. 2016: AICcmodavg: Model Selection and Multimodel Inference Based on (Q)AIC(c). R Package Ver. 2.1-0. URL: https://cran.r-project.org/package=AICcmodavg.

Messina F.J. 1993: Heritability and 'evolvability' of fitness components in Callosobruchus maculatus. - Heredity 71: 623629.

Messina F.J. 1998: Maternal influences on larval competition in insects. In Mousseau T.A. \& Fox C.W. (eds): Maternal Effects as Adaptations. Oxford University Press, New York, pp. 227-243.

Messina F.J. 2004a: How labile are the egg-laying preferences of seed beetles? - Ecol. Entomol. 29: 318-326.

Messina F.J. 2004b: Predictable modification of body size and competitive ability. — Evolution 58: 2788-2797.

Messina F.J. \& DuRham S.L. 2013: Adaptation to a novel host by a seed beetle (Coleoptera: Chrysomelidae: Bruchinae): effect of source population. - Environ. Entomol. 42: 733-742.

Messina F.J. \& DuRham S.L. 2015: Loss of adaptation following reversion suggests trade-offs in host use by a seed beetle. $-J$. Evol. Biol. 28: 1882-1891.

Messina F.J. \& Johnson D. 2014: Specificity of adaptation to a novel host plant by a seed beetle. - Entomol. Exp. Appl. 153: 231-239.

Messina F.J. \& Jones J.C. 2009: Does rapid adaptation to a poorquality host by Callosobruchus maculatus (F.) cause crossadaptation to other legume hosts? - J. Stor. Prod. Res. 45: 215-219.

Messina F.J. \& KarRen M.E. 2003: Adaptation to a novel host modifies host discrimination by the seed beetle Callosobruchus maculatus. - Anim. Behav. 65: 501-507.

Messina F.J. \& Mitchell R. 1989: Intraspecific variation in the egg-spacing behavior of the seed beetle Callosobruchus maculatus. - J. Insect Behav. 2: 727-742. 
Moller H., Smith R.H. \& Sibly R.M. 1990: Evolutionary demography of a bruchid beetle. III. Correlated responses to selection and phenotypic plasticity. - Funct. Ecol. 4: 489-493.

Mousseau T.A. \& RofF D.A. 1987: Natural selection and the heritability of fitness components. - Heredity 59: 181-197.

Nwanze K.F. \& Horber E. 1975: Seed coats of cowpeas affect oviposition and larval development of Callosobruchus maculatus. - Environ. Entomol. 5: 213-218.

Nwanze K.F., Horber E. \& PitTs C.W. 1975: Evidence for ovipositional preference of Callosobruchus maculatus for Cowpea varieties. - Environ. Entomol. 4: 409-412.

ORR H.A. 2005: The genetic theory of adaptation: a brief history. - Nat. Rev. Genet. 6: 119-127.

Risch S.J., Andow D. \& Altieri M.A. 1983: Agroecosystem diversity and pest control: Data, tentative conclusions, and new research directions. - Environ. Entomol. 12: 625-629.

SCRIBER J.M. 2002: Evolution of insect-plant relationships: chemical constraints, coadaptation, and concordance of insect/plant traits. - Entomol. Exp. Appl. 104: 217-235.
SiNGH S.R. 1977: Cowpea cultivars resistant to insect pests in world germplasm collection. - Trop. Grain Legum. Bull. 9: 3-7.

Stastny M., Battisti A., Petrucco-Toffolo E., Schlyter F. \& LARSSON S. 2006: Host-plant use in the range expansion of the pine processionary moth, Thaumetopoea pityocampa. - Ecol. Entomol. 31: 481-490.

Tuda M. 2007: Applied evolutionary ecology of insects of the subfamily Bruchinae (Coleoptera: Chrysomelidae). - Appl. Entomol. Zool. 42: 337-346.

Tuda M., Kagoshima K., Toquenaga Y. \& Arnqvist G. 2014: Global genetic differentiation in a cosmopolitan pest of stored beans: Effects of geography, host-plant usage and anthropogenic factors. - PLOS ONE 9: 1-11.

Wasserman S.S. \& FutuYMa D.J. 1981: Society for the study of evolution. - Evolution 35: 605-617.

Received November 17, 2016; revised and accepted May 9, 2017 Published online May 26, 2017 